\title{
Analysis of Spatial Diversity in Both Transmitter and Receiver on Low-Height Links at Forests
}

\author{
Iñigo Cuiñas, ${ }^{1}$ Paula Gómez-Pérez, ${ }^{2}$ José A. Gay-Fernández, ${ }^{3}$ Javier López, ${ }^{1}$ \\ Diego Pascual, ${ }^{1}$ Laura Rodríguez, ${ }^{1}$ and Marta Muñiz ${ }^{1}$ \\ ${ }^{1}$ Departamento de Teoría do Sinal e Comunicacións, Universidade de Vigo, Vigo, Spain \\ ${ }^{2}$ Centro Universitario de la Defensa, Marín, Spain \\ ${ }^{3}$ Monet Tecnología e Innovación, Nigrán, Spain \\ Correspondence should be addressed to Iñigo Cuiñas; inhigo@uvigo.es
}

Received 9 June 2016; Revised 1 September 2016; Accepted 1 November 2016

Academic Editor: Herve Aubert

Copyright (C) 2016 Iñigo Cuiñas et al. This is an open access article distributed under the Creative Commons Attribution License, which permits unrestricted use, distribution, and reproduction in any medium, provided the original work is properly cited.

\begin{abstract}
This work focuses on radio wave propagation within forested environments, at $5.8 \mathrm{GHz}$. Concretely, we explore the advantages of implementing spatial diversity in reception or even in both ends for improving the strength of the received signal in such environments, which could be useful in applications such as vehicle-to-infrastructure, vehicle-to-vehicle, or emergency communications. Measurements gathered at both evergreen and deciduous forests sustain the thesis. Once processed, the results support the proposal of implementing a spatial diversity technique in reception or in both ends using a $2 \times 4$ (or $2 \times 2$ ) scheme in order to improve the connectivity at $5.8 \mathrm{GHz}$ band within forests. In fact, we estimated a gain due to spatial diversity in reception of $5 \mathrm{~dB}$ and $2 \mathrm{~dB}$ at evergreen and deciduous forests, respectively, and $16 \mathrm{~dB}$ or $5 \mathrm{~dB}$ when implementing at both ends.
\end{abstract}

\section{Introduction}

Propagation within vegetation environments, in general, and forests, in particular, appears as a trending topic in radio communication research, as it focuses on promising areas: those places at which wireless technologies try to expand. Many emerging applications for wireless systems take place in vegetation environments: sensor networks for firing prevention [1], networks for tracking elderly rural people living alone [2], rescue and emergency missions [3], or even vehicle to infrastructure communications [4] would occur in the surroundings of such environments. Those applications make a massive usage of peer-to-peer configurations, in which all transmitter and receiver antennas are installed at low heights (i.e., between 1.5 and 2 meters from ground level), and they are moving from one place to another, as emergency crew carry the antennas as they advance within the scenario. There are fewer researches on these configurations than on typical base station to mobile terminal schemes with base station antennas installed at higher locations. Therefore, having a good knowledge on this specific radio channel behavior would be necessary for radio frequency designers.

This work focuses on the $5.8 \mathrm{GHz}$ band, which is a de facto standard for vehicle to infrastructure communications [5], but it is also useful for other applications among those previously commented on. We take into account its classification as unlicensed band, allocated for radiolocation as primary services and amateur as secondary [6]. The growing number of researches in that band would make us think that everything has been studied at such portion of the spectrum. However, to the knowledge of the authors, few works put the focus on spatial diversity and none on spatial diversity within forest environments.

We based all developments in measured data, gathered at two evergreen environments (a pine tree forest and a eucalyptus forest) and another deciduous forest, considered in both summer and winter seasons. Based on those gathered data, we synthesized receiver spatial diversity systems, computing the gain provided by these additional receiving new branches. Then, we synthesized spatial diversity at both 
ends, in $2 \times 2$ (two transmitting antennas and two receiving antennas) or $2 \times 4$ schemes and also estimated the gain provided. From the obtained figures, we try to answer two key questions: Does spatial diversity $[3,7,8]$ along the surface of the tree provide advantages in peer-to-peer communications within vegetation environments? Is it worth extending the installation of spatial diversity at both radio links ends [9] in forests?

The second section explains the measurement setup and the campaigns designed to gather data from real world. We analyzed the raw data and computed the values governing an exponentially decay law at different forest environments. This analysis is configured in the third section. First, we processed the measured data in order to obtain the receiving spatial diversity performance (one transmitter and two to four receivers) and then the double-end spatial diversity performance when the system works in a $2 \times 2$ or $2 \times 4$ ( 2 transmitters and 2 or 4 receivers) configuration within each of the considered forest environment, described in section four. Finally, section five is devoted to the concluding remarks.

\section{Measurements}

Experimental results support the proposal of this paper. The following paragraphs describe the setup, the procedure, and the environments used during the measurement campaign to gather received power data.

2.1. Measurement Setup. The measurement setup consisted of separate transmitter and receiver. A Rohde-Schwarz SMR40 microwave generator provided pure tones at $5.8 \mathrm{GHz}$ to a RadioMetrics omnidirectional biconical antenna EM6865. The antenna gain is approximately $2.7 \mathrm{dBi}$ and the half powerbeam width is around 40 degrees in elevation. We constructed the receiver end around a Rohde-Schwarz FSP-6 spectrum analyzer, fed by an identical antenna.

We used antennas with omnidirectional azimuth patterns in order to assure that all possible multipath components reached the receiver, as this is the most common situation in applications with nonsophisticated receivers: automatic systems (as car-to-car or car-to-infrastructure communications) or those portable systems carried by emergency staff. Besides, the potential applications of peer-to-peer systems are those that do not allow complex, energy-consuming, and heavy equipment.

2.2. Measurement Environments. Two different evergreen forests and an additional deciduous forest, checked at two opposite seasons, constituted the environments for the measurement campaign. The evergreen environments were a pine tree forest and a eucalyptus forest. The deciduous forest was mainly conformed by oak trees, with some other species. The mean diameter at breast height for pine trees was $32 \mathrm{~cm}$, the eucalyptus measured $44 \mathrm{~cm}$, and the oak trees were $10 \mathrm{~cm}$, as the specimens were younger.

Some additional features of the pine tree woodland are its tree density and the height of the vegetation under the trees (shrubbery, grass, etc.). Pine tree forest has an estimated density of 0.079 pine trees per square meter, and the height of the vegetation under them reached an average value of $93 \mathrm{~cm}$. The eucalyptus forest presented a density of 0.044 eucalyptuses per square meter; and its surrounding vegetation height had an average value of $71 \mathrm{~cm}$. The deciduous forest has a density of 0.062 trees per square meter, and the undertree vegetation height is around $67 \mathrm{~cm}$.

2.3. Measurement Procedure. At each environment, the transmitter was installed close to a reference tree within the forest, whereas the receiver was moved along three radials, centered in such reference tree. At each receiver location, around each tree, and along every radial, four measurement spots were selected: three in line of sight (LoS) conditions (at $0^{\circ}$ with respect to the reference transmission tree, at $90^{\circ}$, and at $270^{\circ}$ ) and an additional one in obstructed LoS (OLoS) conditions (at $180^{\circ}$ respect to the reference transmission tree). In the case of deciduous forests, as the tree trunks were thinner, we only selected two measurement spots around each tree (LoS: $0^{\circ}$ and OLoS: $180^{\circ}$ ). Although we gathered data at each individual receiving locations, we took special care to maintain unchanged the environment conditions, in order to allow us to consider that the environment is the same at all measurement times in different spots at each receiving location.

Besides, two transmission spots were used around the reference transmission tree: one to the side of the tree pointed to the radial under measurement (front transmitter) and the other at the opposite side (back transmitter). Therefore, for each pair of transmitter-receiver location, we had eight (four for deciduous forests) possible combinations of transmitter and receiver spots (2Tx by $4 \mathrm{Rx}$ or 2 by 2 for deciduous forests), which will allow us to perform a synthetic receiver spatial diversity and even double-end spatial diversity analysis.

Figure 1 describes the geometrical disposition of transmitting and receiving antennas at the considered spots at each transmitter/receiver location.

At each combination of transmitter and receiver spots, we configured the spectrum analyzer in a zero-spam mode for gathering 301 samples of received power [10]. After the measurement campaign, up to 250 pairs of transmitterreceiver locations have been tested ( 48 at pine forest, 70 at eucalyptus forest, and 132 at the deciduous forest, where we measured twice, one at each season), each with the eight (or four) radio channel versions. This amount of data provides samples enough to consider the results statistically significant and to confer credibility to the numerical estimations we constructed on these data.

\section{Analysis of Results}

After the measurement campaign, we had a set of series of measured received power data, at different distances, and transmitted and received from/at different spots around each measurement point. Fitting a classical equation allows a simple first evaluation of this data, obtaining a rule similar to standard path loss formula (1) following an exponential law:

$$
p=p_{0} d^{-n}
$$




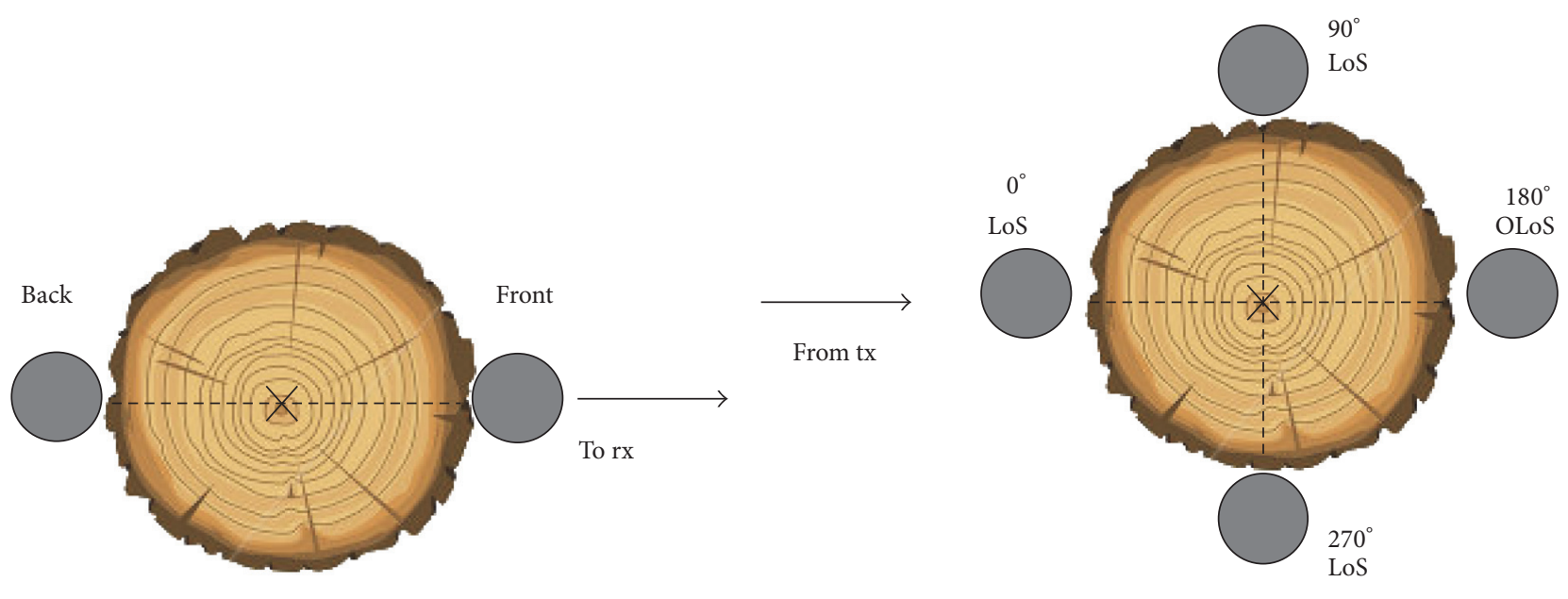

(a)

(b)

FIGURE 1: Geometrical disposition of antenna spots at each measurement location: (a) transmitter end; (b) receiver end.

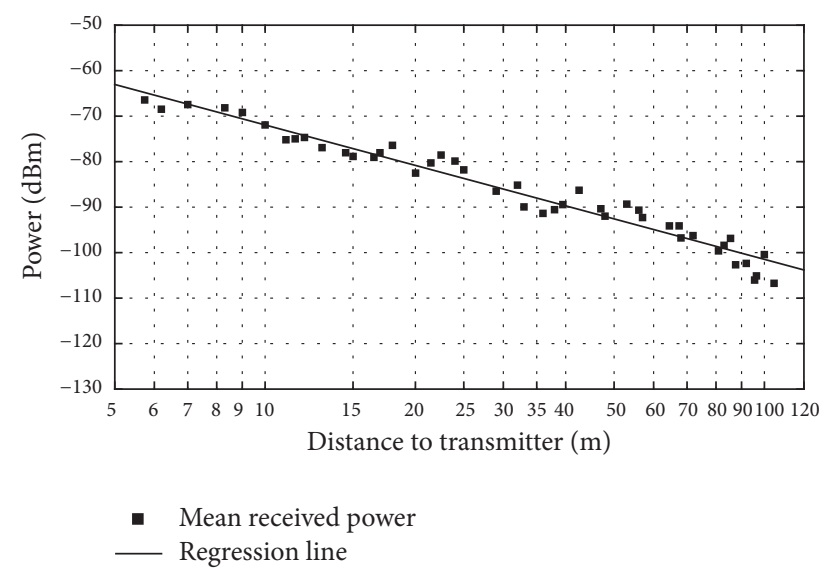

Figure 2: Pine tree, received power $(\mathrm{dBm})$ versus distance in logarithm axis, Tx front, and $\mathrm{Rx}$ angle LoS.

where $p$ is the received power in $\mathrm{mW}$ at a distance $d$ from the transmitter, $p_{0}$ represents the reference power in $\mathrm{mW}$ at 1 meter from the transmitter, and $n$ is the rhythm of the power decay with the distance. Transforming this equation into a logarithmic one, by simply applying logarithms to both sides (1), the following equation comes up:

$$
P=P_{0}-10 n \log _{10}(d) .
$$

Figures 2-5 depict the linear fittings to (2), grouping the measured data by similar conditions: front or back transmitter and receiver in $\operatorname{LoS}\left(0^{\circ}, 90^{\circ}\right.$, and $\left.270^{\circ}\right)$ or in $\operatorname{OLOS}\left(180^{\circ}\right)$, for the case of pine forest. We considered the three data series at each measurement location under LoS condition as we gathered them in the same point.

All radio channels presented similar behavior: the received power monotonously decreases as distance between transmitter and receiver grows. Besides, the rhythm of decay is generally larger than that considered in Friis formula for

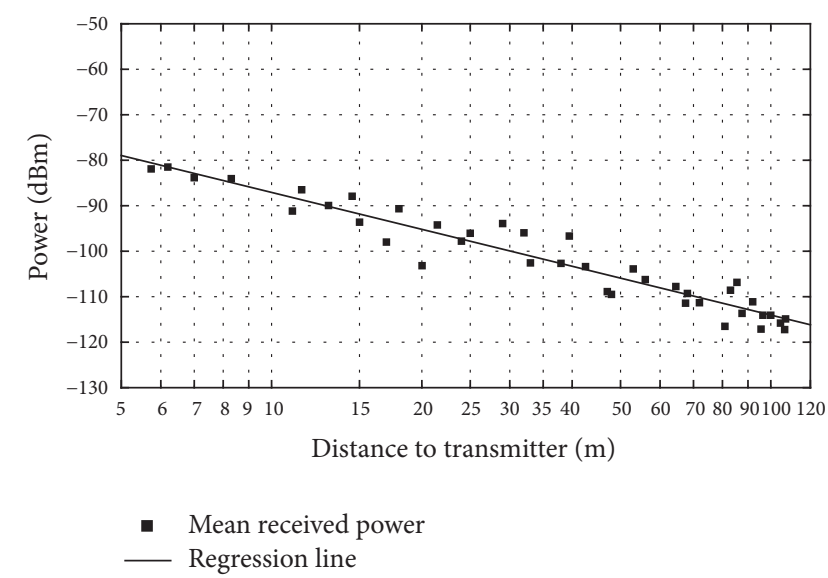

FIGURE 3: Pine tree, received power $(\mathrm{dBm})$ versus distance in logarithm axis, Tx front, and Rx angle OLoS.

free space conditions $(n=2)$, with some exceptions when attenuations at short distances dominate the pattern.

Besides, Tables 1, 2, and 3 summarize the numerical values of each parameter, $P_{0}$ and $n$, for pine, eucalyptus, and oak tree forests, respectively. The path loss exponents were larger than that for theoretical free space conditions $(n=2)$, in almost all situations, which is a logical result since attenuation is supposed to be larger at complex vegetation environments than at open areas.

The only path loss exponent below $n=2$ corresponds to OLoS conditions with transmitter in the back of the transmission reference tree within the pine tree forest. In this case, the initial attenuation induced by the own reference tree is especially deep: the reference power, at 1 meter from the transmitter, is the lowest among all analyzed environments as checked in Tables 1-3. Besides, the absence of a clearly dominant direct ray leads to a reception environment along all radials with many multipath contributions, most of them with similar amplitude. The combination of both situations 


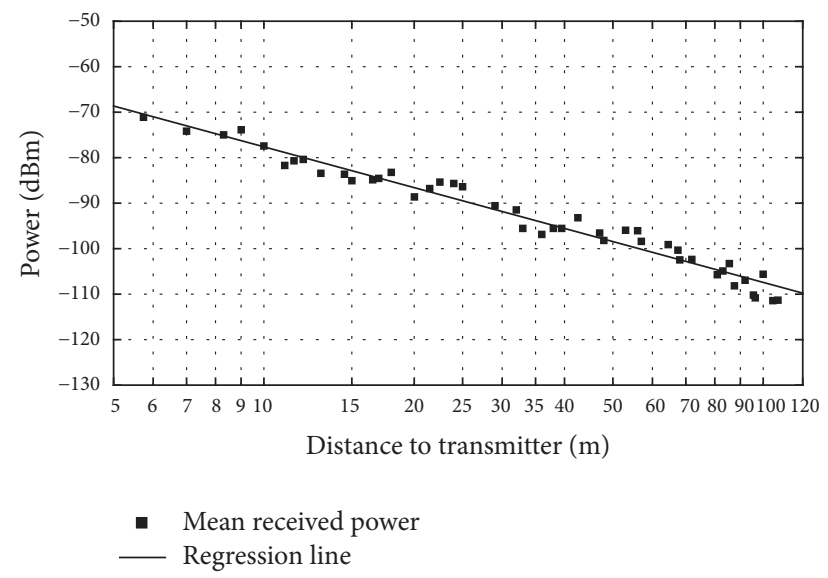

FIgURE 4: Pine tree, received power $(\mathrm{dBm})$ versus distance in logarithm axis, Tx back, and $\mathrm{Rx}$ angle LoS.

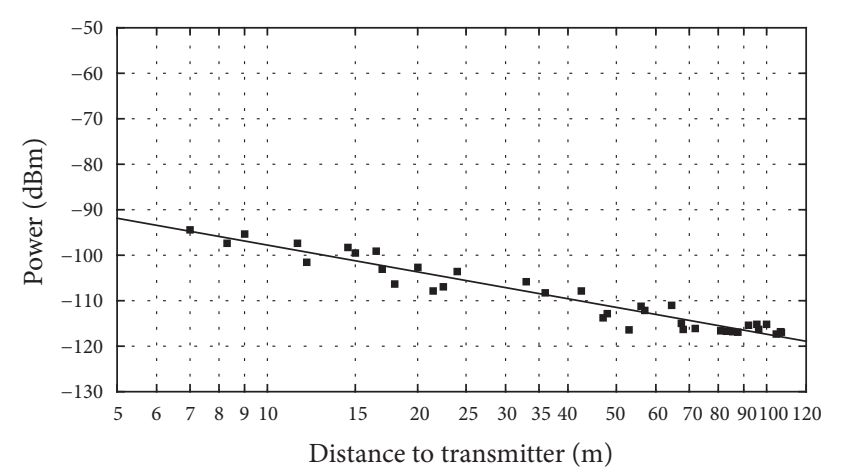

- Mean received power

— Regression line

Figure 5: Pine tree, received power $(\mathrm{dBm})$ versus distance in logarithm axis, Tx back, and $\mathrm{Rx}$ angle OLoS.

TABLE 1: Propagation model parameters, pine forest.

\begin{tabular}{lccc}
\hline \multirow{2}{*}{ Transmitter } & Receiver & \multicolumn{2}{c}{ Parameter } \\
& & $P_{0}(\mathrm{dBm})$ & $n$ \\
\hline \multirow{2}{*}{ Front } & LoS & -42.4 & 2.95 \\
& OLoS & -60.1 & 2.70 \\
\multirow{2}{*}{ Back } & LoS & -47.8 & 2.98 \\
& OLoS & -78.2 & 1.96 \\
\hline
\end{tabular}

forces a strong initial attenuation near the transmitter and a slow decay of attenuation with distance, with an exponent slightly below the free space reference.

The exponents in LoS were larger than in OLoS, probably because the initial attenuation in the last condition (induced by the own reference transmission tree and the trees at short distances and observable at the $P_{0}$ columns in Tables 1-3) dominated the behavior of the environment.
TABLE 2: Propagation model parameters, eucalyptus forest.

\begin{tabular}{lccc}
\hline \multirow{2}{*}{ Transmitter } & Receiver & Parameter & \\
& & $P_{0}(\mathrm{dBm})$ & $n$ \\
\hline \multirow{2}{*}{ Front } & LoS & -46.1 & 2.48 \\
& OLoS & -62.5 & 2.14 \\
\multirow{2}{*}{ Back } & LoS & -54.1 & 2.34 \\
& OLoS & -71.0 & 2.11 \\
\hline
\end{tabular}

TABLE 3: Propagation model parameters, deciduous forests.

\begin{tabular}{lcccc}
\hline \multirow{2}{*}{ Season } & \multirow{2}{*}{ Transmitter } & \multirow{2}{*}{ Receiver } & \multicolumn{2}{c}{ Parameter } \\
& & & $P_{0}(\mathrm{dBm})$ & $n$ \\
\hline \multirow{3}{*}{ Winter } & \multirow{2}{*}{ Front } & LoS & -46.9 & 2.56 \\
& & OLoS & -59.2 & 2.48 \\
& \multirow{2}{*}{ Back } & LoS & -49.1 & 2.86 \\
& & OLoS & -81.8 & 2.74 \\
\hline \multirow{3}{*}{ Summer } & \multirow{2}{*}{ Front } & LoS & -32.1 & 3.66 \\
& \multirow{2}{*}{ Back } & OLoS & -55.8 & 2.84 \\
& & LoS & -41.6 & 2.61 \\
& & OLoS & -80.7 & 2.40 \\
\hline
\end{tabular}

\section{Spatial Diversity Performance}

Using the gathered data, we synthesized both a receiving spatial diversity scheme and a double-end spatial diversity configuration. Commonly, the purpose of diversity is the fading mitigation; that is, this is a technique to mitigate the random fluctuation or change of channels. However, measurements did not show large multipath fading. In fact, the main effect seems to be the antenna pattern distortion and LoS shadowing by the closest tree, which seems to be deterministic. Why then we apply spatial diversity? Although we gathered measured data in a static situation, the target application (emergency communication) will have an intrinsic and unpredictable random component, which is the movement of the staff carrying the antennas and, then, the randomization of the radio channels. This random situation, in actual applications, moved us to check the performance of using spatial diversity along the surface of the tree, as a model of spatial diversity around emergency-personnel bodies (the persons that would carry backpacks with transceivers, with more than one antenna). The next subsections explain the analysis and the estimated performance of such proposals.

4.1. Receiving Spatial Diversity Analysis. Taken into account the measurement procedure, we have two or four measurement spots at each receiving location. We select the maximum value among the two or four mean received powers, considering the transmitter in the most favorable location in terms of propagation, that is, when one expects to obtain less advantage from spatial diversity: the transmitter is in front of the radial under test. Repeating the process for all three radials at every environment, we obtained a set of maximum measured power vectors, and then we compared those to the mean values at each location to compute the spatial diversity gain. This means that we compute the gain we 
TABLE 4: Receiver spatial diversity power gain $(\mathrm{dB})$.

\begin{tabular}{lc}
\hline Environment & Gain $(\mathrm{dB})$ \\
\hline Pine tree forest & 5.6 \\
Eucalyptus forest & 5.3 \\
Deciduous forests-winter & 2.1 \\
Deciduous forests-summer & 1.9 \\
\hline
\end{tabular}

could obtain when implementing spatial diversity, compared to a typical or mean situation when using a simple receiver.

4.2. Double-End Spatial Diversity Analysis. Using the gathered data, we performed a $2 \times 2$ or $2 \times 4$ analysis: for each 250 receiver locations we have eight series of 301 received power samples, transmitted from 2 spots around the reference transmitter tree and received from 2 or 4 spots at the receiver location. Therefore, we can synthesize 250 double-end spatial diversity channels.

Firstly, we compute the median among the 301 received power samples at each of the four or eight branches or subchannels of the static radio channel at each receiver location. As a result, a 2-by-2 or a 2-by-4 matrix of median received powers would represent each of the 250 receiving locations:

$$
P_{i}=\left(\begin{array}{cccc}
P_{f 0, i} & P_{f 90, i} & P_{f 180, i} & P_{f 270, i} \\
P_{b 0, i} & P_{b 90, i} & P_{b 180, i} & P_{b 270, i}
\end{array}\right),
$$

where $i$ represents the receiver location, and the subindexes represent front $(f)$ or back $(b)$ spots at reference transmitter tree, and the angle indicates the spot (LoS: $0^{\circ}, 90^{\circ}$, and $270^{\circ}$; OLoS: $180^{\circ}$ ) at receiver location (i).

Within each matrix, at receiver location $i$, we determine the maximum and the mean received power. Then, we obtained the double-end diversity gain, by comparing maximum and mean received powers, at each receiving location so that we compute the gain we could obtain when implementing double-end spatial diversity, compared to the mean situation when using a simple receiver, which would correspond to a random location of the individual transmitter and receiver of a given radio link.

4.3. Results. As expected, the mere implementation of any spatial diversity technique at the receiver provides a gain with respect to the simple system with only one receiving antenna. Table 4 summarizes the gains given by the use of such technique. Propagation within evergreen forests, which were denser than deciduous, experiments a more important gain over $5 \mathrm{~dB}$. Even in deciduous forests, the spatial diversity gain results are noticeable, around $2 \mathrm{~dB}$.

The larger values of the four-receiver diversity compared to two-receiver are related to the size of the tree trunks: considered evergreen trees were broader than deciduous, and so the OLoS branch at those gives lower received power than the OLoS at these. Then, it leads to larger gains, computed as a comparison between maximum and mean powers among the diversity branches.

Table 5 contains the gains synthesized for double-end diversity implementation. All computed gains are larger than
TABLE 5: Double-end diversity power gain (dB).

\begin{tabular}{lc}
\hline Environment & Gain (dB) \\
\hline Pine tree forest & 16.7 \\
Eucalyptus forest & 16.1 \\
Deciduous forests-winter & 5.0 \\
Deciduous forests-summer & 4.3 \\
\hline
\end{tabular}

those given by spatial diversity, which indicates that the addition of another spot at transmitter location increases the probability of finding a better radio channel for connecting two low-height transceivers for in-forest communications.

We detect that double-end diversity schemes provides gain at all considered locations, related to the mean situation. Maximum gain could reach values over $16 \mathrm{~dB}$ in evergreen environments and over $4 \mathrm{~dB}$ within deciduous environments. This means that double-end spatial diversity implementation multiplies by 3 the gain obtained by just spatial diversity in reception at evergreen forests and by 2 in deciduous forests. We have to highlight that we synthesized a $2 \times 4$ scheme at evergreen forests and a $2 \times 2$ scheme at deciduous forests, due to the nature of the gathered measurements.

The large values are also due to the way of obtaining the gain. It is a comparison between maximum power received by one of the branches and the mean power of all branches at a receiving location. When adding the transmission from the back of the reference tree (blocked by the trunk), the mean received power reduces whereas the maximum is still the same in most of the situations. This is not a trick, as random movement of transceivers in emergency communications could derive in any considered geometric situation.

The behavior of eucalyptus and pine forests seems to be similar, and the numerical values fall within analogous ranges. Besides, the season effect seems to be not so important in the case of deciduous forests, although gain is slightly larger in summer than in winter, independently of the implemented technique.

\section{Conclusions}

We presented and analyzed the results of a large measurement campaign performed within two different evergreen and two season deciduous forest environments. The objective of the campaign was to gather real-world data to analyze and model the radio propagation at $5.8 \mathrm{GHz}$ in complex environments, as a help for possible future applications in designing and deploying radio networks for vehicular or even emergency communications.

The first step in the analysis of measured results was the definition of a simple propagation model for LoS and OLoS conditions. Based on these models, we stated that the rhythm of decay with distance is, generally, larger than that observed at free space conditions.

The first analysis focused on synthesizing spatial diversity implementation at the receiver end. We obtained gains around $5 \mathrm{~dB}$ at evergreen forests and $2 \mathrm{~dB}$ at deciduous forests. 
Then, we tested the performance of a double-end spatial diversity scheme consisted of two transmitters and two or four receivers for each communication link. The gain provided by this synthetic setup was found around $16 \mathrm{~dB}$ and $5 \mathrm{~dB}$ for evergreen and deciduous environments, respectively. A gain has been detected at all locations when applying this proposal, which indicates that the performance of the system would be, in mean, better compared with a traditional SISO (single-input single-output) system.

The decision of implementing or not these spatial diversity proposals will be determined by the losses that the needed processing circuitry will induce when selecting the maximum among the powers received from all two, four, or eight branches or when computing the mean among them. If these losses were significantly below the expected gain, the double-end spatial diversity proposal would be a good solution in such forest environments.

\section{Competing Interests}

The authors declare that they have no competing interests.

\section{Acknowledgments}

This work was partially funded by the Spanish Ministry of Economy and Competitiveness, State Secretary for Research under Project TEC2014-55735-C03-3R, Atlantic Research Centre, the European Regional Development Fund (ERDF), and Xunta de Galicia (Project GRC1015/019). The authors would like to thank Ms. Fernández, Mr. Vázquez, Mr. Marcos, Ms. Pérez, Mr. Deza, Mr. Martínez, Ms. Varela, Ms. Dosil, Mr. Vila, Ms. Rivera, Ms. Rodríguez, Ms. Mar, Ms. Alfonsín, Ms. Roca, Mr. Carlos, Ms. Bey, Mr. González, Mr. Rodríguez, Mr. Canedo, Ms. Abelleira, and Ms. Garrido for their help during the measurement campaigns.

\section{References}

[1] A. G. Colombo and A. L. Vetere Arellano, "Lessons learnt from forest fire disasters," EUR 20662, European Commission, Joint Research Centre, Ispra, Italy, 2003.

[2] N. Blaunstein, D. Censor, and D. Katz, "Radio propagation in rural residential areas with vegetation," Progress in Electromagnetics Research, vol. 40, pp. 131-153, 2003.

[3] I. Cuiñas and J. A. Gay-Fernández, "A proposal on spatial diversity in emergency communications within forest environments," in Proceedings of the 8th European Conference on Antennas and Propagation (EuCAP '14), pp. 1295-1298, The Hague, The Netherlands, April 2014.

[4] I. Sen and D. W. Matolak, "Vehicle-vehicle channel models for the 5-GHz band," IEEE Transactions on Intelligent Transportation Systems, vol. 9, no. 2, pp. 235-245, 2008.

[5] P. E. Ross, “Thus spoke the autobahn," IEEE Spectrum, vol. 52, no. 1, pp. 52-55, 2015.

[6] International Telecommunication Union, "Radio Regulations. Articles," 2012.

[7] C. B. Dietrich Jr., K. Dietze, J. R. Nealy, and W. L. Stutzman, "Spatial, polarization, and pattern diversity for wireless handheld terminals," IEEE Transactions on Antennas and Propagation, vol. 49, no. 9, pp. 1271-1281, 2001.
[8] M. Grabner, V. Kvicera, P. Pechac, and L. Pecha, "Spatial diversity improvement on $10 \mathrm{GHz}$ fixed terrestrial path," in Proceedings of the Loughborough Antennas and Propagation Conference (LAPC '09), pp. 333-336, IEEE, Loughborough, UK, November 2009.

[9] A. J. Paulraj, D. A. Gore, R. U. Nabar, and H. Bölcskei, "An overview of MIMO communications-a key to gigabit wireless," Proceedings of the IEEE, vol. 92, no. 2, pp. 198-217, 2004.

[10] J. A. Gay-Fernández and I. Cuiñas, "Peer to peer wireless propagation measurements and path-loss modeling in vegetated environments," IEEE Transactions on Antennas and Propagation, vol. 61, no. 6, pp. 3302-3311, 2013. 


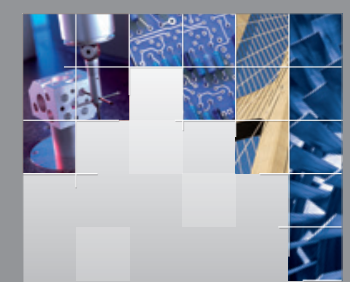

\section{Enfincering}
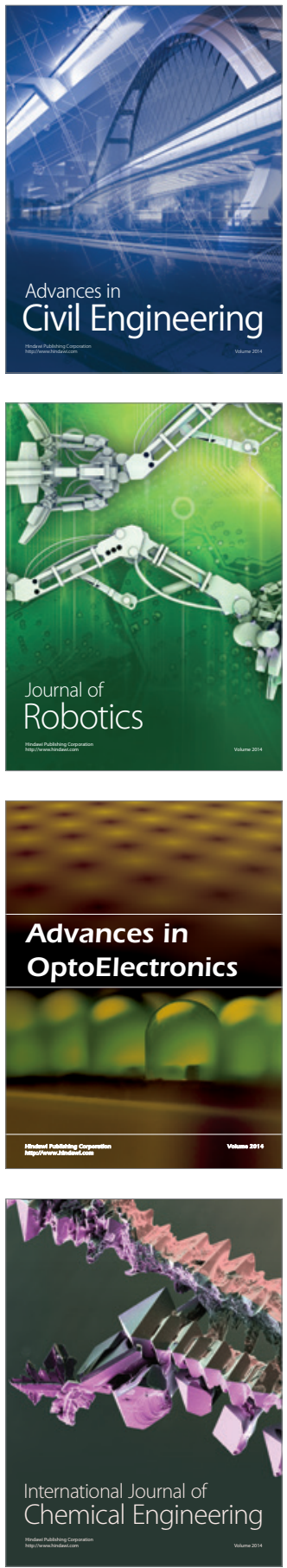

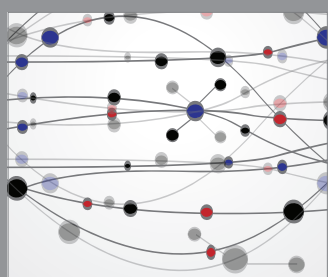

The Scientific World Journal

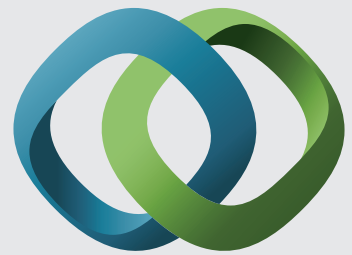

\section{Hindawi}

Submit your manuscripts at

http://www.hindawi.com
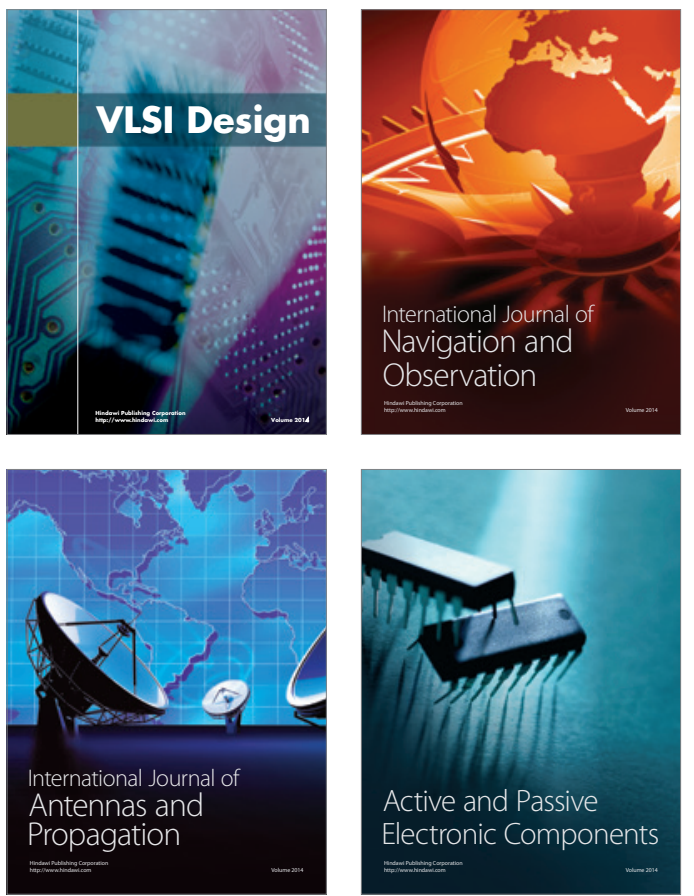
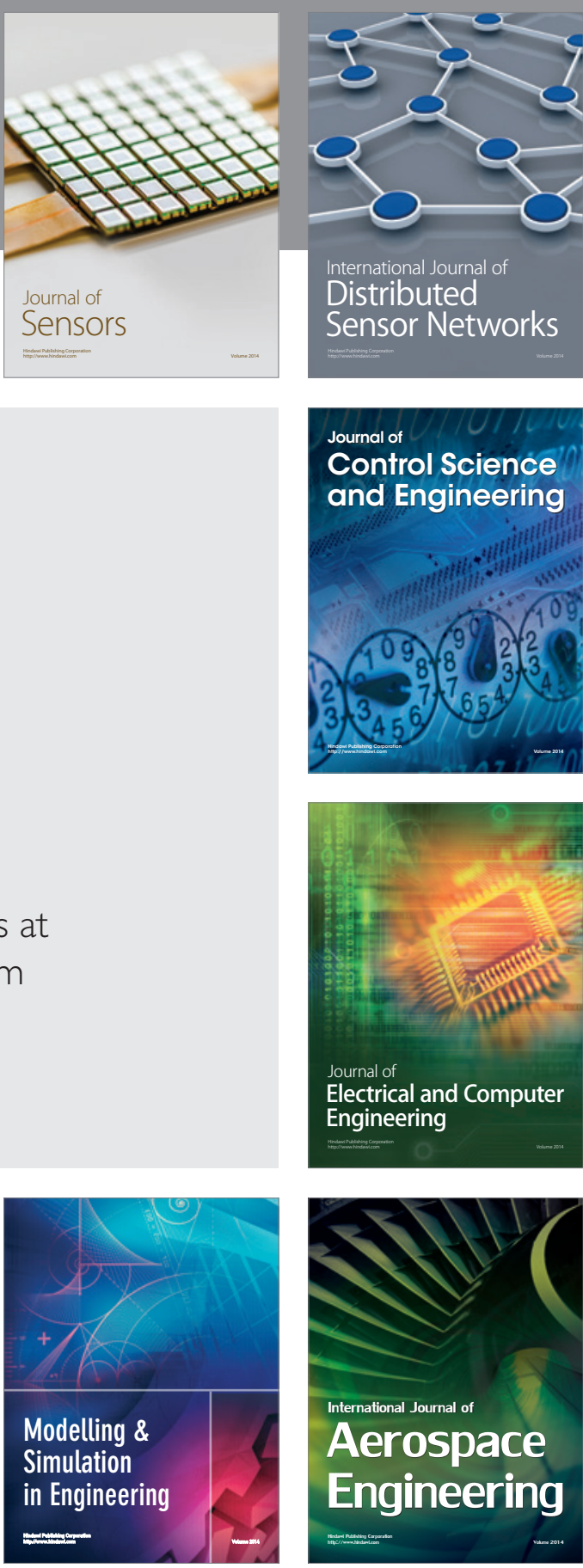

International Journal of

Distributed

Sensor Networks

Journal of

Control Science

and Engineering
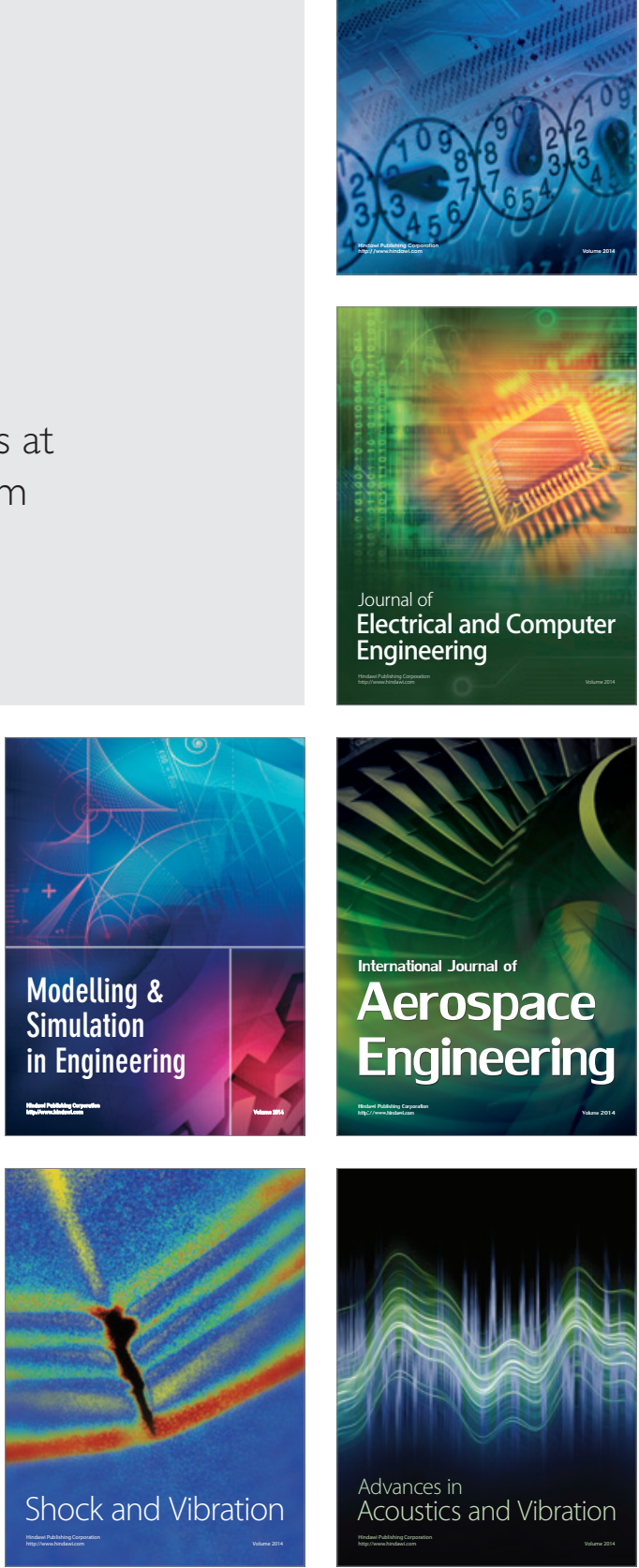